\title{
ON THE INTERSECTION OF RATIONAL TRANSVERSAL SUBTORI
}

\author{
SHAHEEN NAZIR
}

(Received 25 July 2007; accepted 17 October 2007)

Communicated by J. Du

\begin{abstract}
We show that under a suitable transversality condition, the intersection of two rational subtori in an algebraic torus $\left(\mathbb{C}^{*}\right)^{n}$ is a finite group which can be determined using the torsion part of some associated lattice. We also give applications to the study of characteristic varieties of smooth complex algebraic varieties. As an example we discuss A. Suciu's line arrangement, the so-called deleted $B_{3}$-arrangement.
\end{abstract}

2000 Mathematics subject classification: primary 14C21, 14F99, 32S22; secondary 14E05, 14H50.

Keywords and phrases: local system, constructible sheaf, twisted cohomology, characteristic variety, pencil of plane curves.

\section{Introduction}

Let $L$ be a free $\mathbb{Z}$-module of finite rank $n$, and let $A \subset L$ and $B \subset L$ be two primitive sublattices, that is, $A$ and $B$ are subgroups such that

$$
\operatorname{Tors}(L / A)=\operatorname{Tors}(L / B)=0 \text {. }
$$

Consider the associated $\mathbb{C}$-vector spaces

$$
V=L \otimes_{\mathbb{Z}} \mathbb{C}, \quad V_{A}=A \otimes_{\mathbb{Z}} \mathbb{C} \quad \text { and } \quad V_{B}=B \otimes_{\mathbb{Z}} \mathbb{C} .
$$

Let $\exp _{L}: V \rightarrow T=L \otimes_{\mathbb{Z}} \mathbb{C}^{*}$ be the associated exponential map given by

$$
\exp _{L}=1_{L} \otimes_{\mathbb{Z}} \exp
$$

where $1_{L}: L \rightarrow L$ is the identity and $\exp : \mathbb{C} \rightarrow \mathbb{C}^{*}$ is defined by $t \mapsto \exp (2 \pi i t)$. Then $\exp _{L}$ is a surjective group homomorphism with kernel $L=L \otimes_{\mathbb{Z}} \mathbb{Z} \subset V$. If a $\mathbb{Z}$-basis

(C) 2009 Australian Mathematical Society 1446-7887/2009 \$16.00 
of $L$ is chosen, then the identifications $L=\mathbb{Z}^{n}, V=\mathbb{C}^{n}, T=\left(\mathbb{C}^{*}\right)^{n}$ are obvious and $\exp _{L}: \mathbb{C}^{n} \rightarrow\left(\mathbb{C}^{*}\right)^{n}$ is given by

$$
\left(t_{1}, \ldots, t_{n}\right) \mapsto\left(\exp \left(2 \pi i t_{1}\right), \ldots, \exp \left(2 \pi i t_{n}\right)\right) .
$$

The main result of this note is the following theorem.

THEOREM 1.1. If $V_{A} \cap V_{B}=0$, then there is a group isomorphism

$$
\operatorname{Tors}(L /(A+B)) \rightarrow \exp _{L}\left(V_{A}\right) \cap \exp _{L}\left(V_{B}\right) .
$$

In fact any algebraic subtorus $S \subset T$, that is, $S$ is a closed algebraic subset and a subgroup in $T$, comes from a primitive lattice $A(S) \subset L$ (see Arapura's paper [1, Lemma 2.1 in Section II]). Hence, Theorem 1.1 applies to any pair of such algebraic subtori.

This theorem is proved in Section 2. In Section 3 we show how to use Theorem 1.1 to describe the intersections of the irreducible components of the characteristic varieties of smooth complex algebraic varieties. A specific example coming from hyperplane arrangement theory concludes the paper.

\section{The proof}

Let $n=\operatorname{rank} L, a=\operatorname{rank} A$ and $b=\operatorname{rank} B$. Consider the quotient group $L^{\prime}=$ $L / A$, which is again a lattice, of rank $n-a$. The composition $B \rightarrow L \rightarrow L^{\prime}$ of the inclusion $B \rightarrow L$ and the projection $L \rightarrow L^{\prime}$ gives rise to an injective morphism $\iota: B \rightarrow L^{\prime}$ identifying $B$ to the sublattice $B^{\prime}=\iota(B) \subset L^{\prime}$.

Then there is a basis $e_{1}^{\prime}, \ldots, e_{n-a}^{\prime}$ of the lattice $L^{\prime}$ such that $B^{\prime}$ is the subgroup spanned by $d_{1} e_{1}^{\prime}, \ldots, d_{b} e_{b}^{\prime}$ for some positive integers $d_{j}$. Moreover, there is an integer $m$ with $1 \leq m \leq b+1$ such that

$$
1=d_{1}=\cdots=d_{m-1}<d_{m} \leq \cdots \leq d_{b} \quad \text { and } \quad d_{m}\left|d_{m+1}\right| \cdots \mid d_{b} .
$$

It follows that

$$
\operatorname{Tors}(L /(A+B))=\operatorname{Tors}\left(\frac{L / A}{(A+B) / A}\right)=\operatorname{Tors}\left(L^{\prime} / B^{\prime}\right)
$$

and hence

$$
\operatorname{Tors}(L /(A+B))=\mathbb{Z} / d_{m} \mathbb{Z} \oplus \mathbb{Z} / d_{m+1} \mathbb{Z} \oplus \cdots \oplus \mathbb{Z} / d_{b} \mathbb{Z}
$$

Let $e_{1}, \ldots, e_{n-a}$ be any lifts of the vectors $e_{j}^{\prime}$ to $L$ and let $f_{1}, \ldots, f_{a}$ be a $\mathbb{Z}$-basis of $A$. Then $\mathcal{B}=\left\{e_{1}, \ldots, e_{n-a}, f_{1}, \ldots, f_{a}\right\}$ is a $\mathbb{Z}$-basis of $L$.

For $j=1, \ldots, b$, let $g_{j} \in B$ be vectors such that their classes $g_{j}^{\prime}$ in $L^{\prime}$ satisfy $g_{j}^{\prime}=d_{j} e_{j}^{\prime}$. It follows that $g_{j}=d_{j} e_{j}+a_{j}$ for some vectors $a_{j} \in A$. Now write

$$
a_{j}=\sum_{i=1, a} \alpha_{j i} f_{i}
$$


for some $\alpha_{j i} \in \mathbb{Z}$. By replacing $e_{j}$ by $e_{j}+r_{j}$ for suitable vectors $r_{j} \in A$, we may and do assume throughout the remainder of this paper that

$$
0 \leq \alpha_{j i}<d_{j}
$$

for all $i=1, \ldots, a$ and $j=1, \ldots, b$. In particular, $a_{j}=0$ for $j=1, \ldots, m-1$.

LEMMA 2.1. The vectors $g_{1}, \ldots, g_{b}$ form a $\mathbb{Z}$-basis of the lattice $B$.

PROOF. Note that the vectors $g_{1}, \ldots, g_{b}$ are all contained in $B$ and their images under $\iota$ span the lattice $B^{\prime}$.

Assume now that $\exp _{L}\left(v_{A}\right)=\exp _{L}\left(v_{B}\right)$ for some vectors

$$
v_{A}=p_{1} f_{1}+\cdots+p_{a} f_{a} \in V_{A}
$$

and

$$
v_{B}=q_{1} g_{1}+\cdots+q_{b} g_{b} \in V_{B}
$$

where $p_{i}, q_{j} \in \mathbb{C}$. It follows that $v_{A}-v_{B} \in \operatorname{ker} \exp _{L}=L$. More precisely, we obtain

$$
q_{j} d_{j} \in \mathbb{Z} \quad \text { for } j=1, \ldots, b
$$

and

$$
z_{i}:=p_{i}-\sum_{j=1, b} q_{j} \alpha_{j i} \in \mathbb{Z} \quad \text { for } i=1, \ldots, a .
$$

It follows that $q_{j}=k_{j} / d_{j}$. We may and do assume that $0 \leq k_{j}<d_{j}$, since the value of $\exp _{L}\left(v_{B}\right)$ is not changed when the coefficients $q_{j}$ are modified by integers. Note that with this choice one has $k_{j}=0$ for $j=1, \ldots, m-1$. In this way we obtain a surjective group homomorphism

$$
\theta: \mathbb{Z} / d_{m} \mathbb{Z} \oplus \mathbb{Z} / d_{m+1} \mathbb{Z} \oplus \cdots \oplus \mathbb{Z} / d_{b} \mathbb{Z} \rightarrow \exp _{L}\left(V_{A}\right) \cap \exp _{L}\left(V_{B}\right)
$$

given by

$$
\hat{k}=\left(\hat{k}_{m}, \ldots, \hat{k}_{b}\right) \mapsto \exp _{L}\left(\frac{k_{m}}{d_{m}} g_{m}+\cdots+\frac{k_{b}}{d_{b}} g_{b}\right) .
$$

This morphism $\theta$ is indeed defined correctly since for any choice of the $q_{j}$ as above we may use the defining equation of $z_{i}$, set $z_{i}=0$ and determine the values for $p_{i}$, that is, find a vector $v_{A}$ such that $\exp _{L}\left(v_{A}\right)=\exp _{L}\left(v_{B}\right)$.

To show that $\theta$ is injective, we have to show that $\operatorname{ker} \theta=0$. Since $B$ is primitive we can take the set

$$
\left\{g_{1}, \ldots, g_{b}, h_{1}, \ldots, h_{n-b}\right\}
$$


as a $\mathbb{Z}$-basis of $L$, where $h_{1}^{\prime}, \ldots, h_{n-b}^{\prime}$ is a $\mathbb{Z}$-basis for the lattice $L / B$. Let $\hat{k} \in \operatorname{ker} \theta$. Then

$$
\theta(\hat{k})=\exp _{L}\left(\frac{k_{m}}{d_{m}} g_{m}+\cdots+\frac{k_{b}}{d_{b}} g_{b}\right)=1,
$$

which implies that $\left(\left(k_{i}\right) / d_{i}\right) \in \mathbb{Z}$, for all $m \leq i \leq b$. Therefore,

$$
\hat{k}=\left(\hat{k}_{m}, \ldots, \hat{k}_{b}\right)=(\hat{0}, \ldots, \hat{0})
$$

that is, $\operatorname{ker} \theta=0$.

\section{On the intersection of irreducible components of characteristic varieties}

3.1. Local systems, characteristic and resonance varieties Let $M$ be a quasiprojective smooth complex algebraic variety. The rank-one local systems on $M$ are parameterized by the algebraic group

$$
\mathbb{T}(M)=\operatorname{Hom}\left(H_{1}(M), \mathbb{C}^{*}\right) .
$$

The connected component $\mathbb{T}^{0}(M)$ of the unit element $1 \in \mathbb{T}(M)$ is an algebraic torus, that is, it is isomorphic to $\left(\mathbb{C}^{*}\right)^{n}$, where $n \in \mathbb{N}$ is the first Betti number of $M$, that is, $n=b_{1}(M)$. It is clear that $\mathbb{T}^{0}(M)=\mathbb{T}(M)$ if and only if the integral homology group $H_{1}(M)$ is torsion free. For $\rho \in \mathbb{T}(M)$, we denote the corresponding local system on $M$ by $\mathcal{L}_{\rho}$.

The computation of the twisted cohomology groups $H^{j}\left(M, \mathcal{L}_{\rho}\right)$ is one of the major problems in many areas of topology. To study these cohomology groups, one idea is to study the characteristic varieties defined by

$$
\mathcal{V}_{m}^{j}(M)=\left\{\rho \in \mathbb{T}(M) \mid \operatorname{dim} H^{j}\left(M, \mathcal{L}_{\rho}\right) \geq m\right\} .
$$

To simplify the notation, we set $\mathcal{V}_{m}(M)=\mathcal{V}_{m}^{1}(M)$. It is known that the following theorem holds, see Beauville [2] and Simpson [12] in the proper case and Arapura [1] in the quasi-projective case.

THEOREM 3.2. The positive-dimensional irreducible components of $\mathcal{V}_{m}(M)$ are subtori in $\mathbb{T}(M)$ translated by elements of finite order. More precisely, for each positive-dimensional irreducible component $W$ of $\mathcal{V}_{m}(M)$ we can write $W=\rho$. $f^{*}(\mathbb{T}(S))$, where $f: M \rightarrow S$ is a surjective regular mapping to a curve $S$ having a connected general fiber and $\rho \in \mathbb{T}(M)$ is a finite-order character.

If $1 \in W$, then we can take $\rho=1$ in the above equality. Let $T_{1} W$ denote the tangent space to $W$ at the identity 1 in such a case. The following theorem is taken from [6, Theorem 2, (b)].

THEOREM 3.3. Let $M$ be a quasi-projective smooth complex algebraic variety. Let $W_{1}$ and $W_{2}$ be two distinct irreducible components of the characteristic variety $\mathcal{V}_{1}(M)$ such that $1 \in W_{1} \cap W_{2}$. Then $T_{1} W_{1} \cap T_{1} W_{2}=0$. 
Note that any such tangent space $T_{1} W \subset H^{1}(M, \mathbb{C})$ is rationally defined, that is, there is a primitive lattice $L \subset H^{1}(M, \mathbb{Z})$ such that $T_{1} W=L \otimes_{\mathbb{Z}} \mathbb{C}$ under the identification

$$
H^{1}(M, \mathbb{C})=H^{1}(M, \mathbb{Z}) \otimes_{\mathbb{Z}} \mathbb{C} .
$$

Indeed, one can take $L$ to be the primitive sublattice $f^{*}\left(H^{1}(S, \mathbb{Z})\right)$, in view of the functoriality of the exponential mapping

$$
\exp : T_{1} \mathbb{T}(M)=H^{1}(M, \mathbb{C}) \rightarrow \mathbb{T}(M)
$$

and of the following lemma.

LEMMA 3.4. Let $f: M \rightarrow S$ be a surjective regular mapping to a curve $S$, having a connected general fiber. Then $f^{*}\left(H^{1}(S, \mathbb{Z})\right)$ is a primitive sublattice in $H^{1}(M, \mathbb{Z})$.

PROOF. Under these conditions, it is well known that the morphism

$$
f_{*}: H_{1}(M, \mathbb{Z}) \rightarrow H_{1}(S, \mathbb{Z})
$$

is surjective. Let $L_{0}$ be a primitive sublattice in $H_{1}(M, \mathbb{Z})$ such that

$$
H_{1}(M, \mathbb{Z})=\operatorname{ker} f_{*} \oplus L_{0} .
$$

Then $f^{*}\left(H^{1}(S, \mathbb{Z})\right)$ can be identified with the dual

$$
L_{0}^{\vee}=\operatorname{Hom}\left(L_{0}, \mathbb{Z}\right)=\left\{u \in \operatorname{Hom}\left(H_{1}(M, \mathbb{Z}), \mathbb{Z}\right) ;\left.u\right|_{\text {ker } f_{*}}=0\right\} .
$$

Moreover

$$
H^{1}(M, \mathbb{Z})=H_{1}(M, \mathbb{Z})^{\vee}=\left(\operatorname{ker} f_{*}\right)^{\vee} \oplus L_{0}^{\vee},
$$

which completes the proof of the claim.

Applying Theorem 1.1 to this setting, we obtain the following corollary.

Corollary 3.5. Let $W_{1}$ and $W_{2}$ be two distinct irreducible components of the characteristic variety $\mathcal{V}_{1}(M)$ such that $1 \in W_{1} \cap W_{2}$. Let $L_{1}$ and $L_{2}$ be the primitive sublattices in $H^{1}(M, \mathbb{Z})$ associated to $W_{1}$ and $W_{2}$, respectively, by the above construction. Then there is a group isomorphism

$$
\operatorname{Tors}\left(H^{1}(M, \mathbb{Z}) /\left(L_{1}+L_{2}\right)\right)=W_{1} \cap W_{2} .
$$

REMARK 3.6. Let $W_{1}$ and $W_{2}$ be two distinct irreducible components of the characteristic variety $\mathcal{V}_{1}(M)$, where at least one of them, say $W_{1}$, is translated, that is, $1 \notin W_{1}$, and that meet at a point $\rho$. Then we may write $W_{1}=\rho \cdot W_{1}^{\prime}$ and $W_{2}=\rho \cdot W_{2}^{\prime}$, where $W_{j}^{\prime}$ are subtori in $\mathbb{T}(M)$.

Assume that $\operatorname{dim} W_{j}>1$ for $j=1,2$ (the claim is obvious when one of the two components is one dimensional) and that $M$ is a hyperplane arrangement 
complement. Then $T_{1} W_{1}^{\prime} \cap T_{1} W_{2}^{\prime}=0$, since $W_{1}^{\prime}$ and $W_{2}^{\prime}$ are again two distinct irreducible components of the characteristic variety $\mathcal{V}_{1}(M)$, see [5]. Moreover, the tangent spaces $T_{1} W_{j}^{\prime}$ are rationally defined and the above corollary yields a set bijection

$$
\operatorname{Tors}\left(H^{1}(M, \mathbb{Z}) /\left(L_{1}^{\prime}+L_{2}^{\prime}\right)\right)=W_{1} \cap W_{2}
$$

where $L_{j}^{\prime}$ is the primitive sublattice associated to $W_{j}^{\prime}$ by the above construction.

Note that any character in such an intersection $W_{1} \cap W_{2}$ has finite order. Indeed, let $W_{1}=\rho_{1} \cdot W_{1}^{\prime}$ and $W_{2}=\rho_{2} \cdot W_{2}^{\prime}$, where $\rho_{1}$ and $\rho_{2}$ are finite-order characters, that is, $\rho_{1}^{m_{1}}=1$ and $\rho_{2}^{m_{2}}=1$. Then $\rho \in W_{1} \cap W_{2}$ implies that $\rho=\rho_{1} w_{1}=\rho_{2} w_{2}$, where $w_{j} \in W_{j}^{\prime}$. Let $m=\operatorname{lcm}\left(m_{1}, m_{2}\right)$. Then $\rho^{m}=\rho_{1}^{m} w_{1}^{m}$ and $\rho^{m}=\rho_{2}^{m} w_{2}^{m}$ which implies that

$$
\rho^{m}=w_{1}^{m}=w_{1}^{m} \Rightarrow \rho^{m} \in W_{1}^{\prime} \cap W_{2}^{\prime}
$$

so, by Corollary 3.5, $\rho^{m}$ is of finite order. Thus, $\rho$ is a finite-order character.

A completely different proof of the finiteness of the intersection $W_{1} \cap W_{2}$ of two distinct irreducible components of the first characteristic variety was given in [7].

Let $H^{*}(M, \mathbb{C})$ be the cohomology algebra of the variety $M$ with $\mathbb{C}$-coefficients. Right multiplication (cup-product) by an element $z \in H^{1}(M, \mathbb{C})$ yields a cochain complex $\left(H^{*}(M, \mathbb{C}), \mu_{z}\right)$. The resonance varieties of $M$ are the jumping loci for the cohomology of this complex, namely

$$
\mathcal{R}_{m}^{j}(M)=\left\{z \in H^{1}(M, \mathbb{C}) \mid \operatorname{dim} H^{j}\left(H^{*}(M, \mathbb{C}), \mu_{z}\right) \geq m\right\} .
$$

To simplify the notation, we set $\mathcal{R}_{m}(M)=\mathcal{R}_{m}^{1}(M)$.

One of the main results relating the characteristic and resonance varieties is the following theorem.

THEOREM 3.7. Let $M$ be a hypersurface arrangement complement. The map exp: $H^{1}(M, \mathbb{C}) \rightarrow \mathbb{T}^{0}(M)$ induces, for any $m, j \geq 1$,

$$
\left(\mathcal{R}_{m}^{j}(M), 0\right) \simeq\left(\mathcal{V}_{m}^{j}(M), 1\right)
$$

This equality of germs implies that the resonance variety $\mathcal{R}_{m}^{j}(M)$ is exactly the tangent cone at one of the characteristic varieties $\mathcal{V}_{m}^{j}(M)$, a fact established by Cohen and Suciu [4] and which can also be derived from [8]. (See also [3, Theorem 3.7].) It was claimed by Libgober that this property holds for any smooth quasi-projective variety [9] but now there are counter-examples to this claim [6].

Remark 3.8. According to Arapura [1, Theorem 1.1 in Section V], under the assumption that $H^{1}(M, \mathbb{Q})$ has a pure Hodge structure, the positive-dimensional irreducible components of all characteristic varieties $\mathcal{V}_{m}^{j}(M)$ are (translated) subtori. Our Theorem 1.1 applies to this more general setting as well. The major difference 
with the case of first characteristic varieties is that distinct components do not necessarily meet only at the origin. In the following we give an example, for which we are grateful to Professor A. Suciu.

Consider the central hyperplane arrangement in $\mathbb{C}^{4}$ defined by the equation

$$
x y z w(x+y+z)(y-z+w)=0 .
$$

Then the corresponding resonance variety $\mathcal{R}_{1}^{2}(M)$ has two three-dimensional components, say $E_{1}$ and $E_{2}$, defined respectively by the ideals

$$
I_{1}=\left(x_{1}+x_{2}+x_{3}+x_{6}, x_{4}, x_{5}\right)
$$

and

$$
I_{2}=\left(x_{1}, x_{2}+x_{3}+x_{4}+x_{5}, x_{6}\right) .
$$

These two components intersect in the line $D=\left(x_{1}, x_{2}+x_{3}, x_{4}, x_{5}, x_{6}\right)$. This implies that the irreducible components $W_{1}=\exp \left(E_{1}\right)$ and $W_{2}=\exp \left(E_{2}\right)$ of the characteristic variety $\mathcal{V}_{1}^{2}(M)$ intersect along the one-dimensional subtorus $\exp (D)$.

The fact that $M$ has a simply connected compactification implies the following.

COROLlaRY 3.9. The irreducible components of $\mathcal{R}_{1}(M)$ are precisely the maximal linear subspaces $E \subset H^{1}(M, \mathbb{C})$, isotropic with respect to the cup product on $M$

$$
\cup: H^{1}(M, \mathbb{C}) \times H^{1}(M, \mathbb{C}) \rightarrow H^{2}(M, \mathbb{C})
$$

PROOF. Let $E$ be a component of $\mathcal{R}_{1}(M)$. By the above theorem there is a component $W$ in $\mathcal{V}_{1}(M)$ such that $1 \in W$ and $T_{1} W=E$. By Arapura's results in [1] we can write $W=f_{E}^{*}(\mathbb{T}(S))$, where $f_{E}: M \rightarrow S$ is a regular mapping to a curve $S$. (Such a mapping $f_{E}$ is said to be associated with the subspace $E$.) Since in our case $S$ is rational, $T_{1} W=f_{E}^{*}\left(H^{1}(S, \mathbb{C})\right)$ is isotropic with respect to the cup product, since the cup product on $H^{1}(S, \mathbb{C})$ is trivial. Maximality of $E$ comes from the fact that $E$ is a component of $\mathcal{R}_{1}(M)$. The restriction $\operatorname{dim} E \geq 2$ comes from [1].

3.2. An example: the deleted $B_{3}$-arrangement Let $\mathcal{A}$ be the deleted $B_{3}$-arrangement which is obtained from the $B_{3}$ reflection arrangement by deleting the plane $x+y-z=0$. A defining polynomial for $\mathcal{A}$ is

$$
Q=x y z(x-y)(x-z)(y-z)(x-y-z)(x-y+z) .
$$

The decone $d \mathcal{A}$ is obtained by setting $z=1$. Let

$$
\begin{gathered}
L_{1}: \ell_{1}=x=0, \quad L_{2}: \ell_{2}=y=0, \quad L_{3}: \ell_{3}=x-y=0, \quad L_{4}: \ell_{4}=x-1=0, \\
L_{5}: \ell_{5}=y-1=0, \quad L_{6}: \ell_{6}=x-y-1=0, \quad L_{7}: \ell_{7}=x-y+1=0
\end{gathered}
$$

be the lines of the associated affine arrangement in $\mathbb{A}^{2}$. Let $L_{8}: \ell_{8}=z=0$ be the line at infinity and let $M$ be the complement of $d \mathcal{A}$ in $\mathbb{A}^{2}$. The resonance variety $R_{1}(d \mathcal{A})$ 
has 12 irreducible components of dimension two and three. These components $E$ and their associated maps $f_{E}: M \rightarrow S$ are given below, see $[13,14]$. Denote by $e_{1}, \ldots, e_{7}$ the $\mathbb{Z}$-basis of $H^{1}(M, \mathbb{Z})$ given by

$$
e_{j}=\frac{1}{2 \pi i} \frac{d \ell_{j}}{\ell_{j}}
$$

see [11]. Then each of the components $E$ is the $\mathbb{C}$-vector space spanned by a primitive lattice denoted by $E^{0}$, that is, $E=E^{0} \otimes_{\mathbb{Z}} \mathbb{C}$, so it is enough in each case to indicate a $\mathbb{Z}$-basis of $E^{0}$.

(1) The local components There are seven local components, corresponding to six triple points and one quadruple point.

(i) For the triple $L_{1} \cap L_{2} \cap L_{3}$,

$$
E_{1}^{0}=\left\langle e_{1}-e_{3}, e_{2}-e_{3}\right\rangle \quad \text { and } \quad f_{E_{1}}(x, y)=\frac{x}{y},
$$

where $S=\mathbb{C} \backslash\{0,1\}$.

(ii) For the triple $L_{3} \cap L_{4} \cap L_{5}$,

$$
E_{2}^{0}=\left\langle e_{4}-e_{5}, e_{4}-e_{3}\right\rangle \quad \text { and } \quad f_{E_{2}}(x, y)=\frac{x-1}{y-1},
$$

where $S=\mathbb{C} \backslash\{0,1\}$.

(iii) For the triple $L_{2} \cap L_{4} \cap L_{6}$,

$$
E_{3}^{0}=\left\langle e_{4}-e_{2}, e_{6}-e_{2}\right\rangle \quad \text { and } \quad f_{E_{3}}(x, y)=\frac{x-1}{y},
$$

where $S=\mathbb{C} \backslash\{0,1\}$.

(iv) For the triple $L_{1} \cap L_{5} \cap L_{7}$,

$$
E_{4}^{0}=\left\langle e_{1}-e_{7}, e_{5}-e_{1}\right\rangle \quad \text { and } \quad f_{E_{4}}(x, y)=\frac{x}{y-1},
$$

where $S=\mathbb{C} \backslash\{0,1\}$.

(v) For the triple $L_{1} \cap L_{4} \cap L_{8}$,

$$
E_{5}^{0}=\left\langle e_{1}, e_{4}\right\rangle \text { and } f_{E_{6}}(x, y)=x,
$$

where $S=\mathbb{C} \backslash\{0,1\}$.

(vi) For the triple $L_{2} \cap L_{5} \cap L_{8}$,

$$
E_{6}^{0}=\left\langle e_{5}, e_{2}\right\rangle \text { and } f_{E_{7}}(x, y)=y,
$$

where $S=\mathbb{C} \backslash\{0,1\}$.

(vii) For the quadruple $L_{3} \cap L_{6} \cap L_{7} \cap L_{8}$,

$$
E_{7}^{0}=\left\langle e_{3}, e_{6}, e_{7}\right\rangle \text { and } f_{E_{5}}(x, y)=x-y,
$$

where $S=\mathbb{C} \backslash\{0, \pm 1\}$. 
(2) The non-local components There are five non-local components, corresponding to braid subarrangements.

(viii) For $X=\left(L_{1} L_{6}\left|L_{3} L_{4}\right| L_{2} L_{8}\right)$,

$$
E_{8}^{0}=\left\langle e_{1}-e_{3}-e_{4}+e_{6}, e_{2}-e_{3}-e_{4}\right\rangle \quad \text { and } \quad f_{E_{8}}(x, y)=\frac{x(x-y-1)}{(x-y)(x-1)},
$$

where $S=\mathbb{C} \backslash\{0,1\}$.

(ix) For $Y=\left(L_{4} L_{8}\left|L_{2} L_{3}\right| L_{5} L_{6}\right)$,

$$
E_{9}^{0}=\left\langle-e_{2}-e_{3}+e_{5}+e_{6}, e_{2}+e_{3}-e_{4}\right\rangle \quad \text { and } \quad f_{E_{9}}(x, y)=\frac{x-1}{y(x-y)},
$$

where $S=\mathbb{C} \backslash\{0,1\}$.

(x) For $Z=\left(L_{1} L_{5}\left|L_{2} L_{4}\right| L_{3} L_{8}\right)$,

$$
E_{10}^{0}=\left\langle e_{1}-e_{2}-e_{4}+e_{5}, e_{2}-e_{3}+e_{4}\right\rangle \quad \text { and } \quad f_{E_{10}}(x, y)=\frac{x(y-1)}{y(x-1)},
$$

where $S=\mathbb{C} \backslash\{0,1\}$.

(xi) For $W=\left(L_{1} L_{3}\left|L_{4} L_{7}\right| L_{5} L_{8}\right)$,

$E_{11}^{0}=\left\langle e_{1}+e_{3}-e_{5}, e_{5}-e_{7}-e_{4}\right\rangle \quad$ and $\quad f_{E_{11}}(x, y)=\frac{x(x-y)}{(x-y+1)(x-1)}$,

where $S=\mathbb{C} \backslash\{0,1\}$.

(xii) For $V=\left(L_{1} L_{8}\left|L_{2} L_{7}\right| L_{3} L_{5}\right)$,

$$
E_{12}^{0}=\left\langle e_{1}-e_{2}-e_{7}, e_{3}+e_{5}-e_{2}-e_{7}\right\rangle \quad \text { and } \quad f_{E_{12}}(x, y)=\frac{x}{y(x-y+1)},
$$

where $S=\mathbb{C} \backslash\{0,1\}$.

One way to obtain these 12 irreducible components $E_{j}$ is to compute the cupproduct

$$
H^{1}(M, \mathbb{C}) \times H^{1}(M, \mathbb{C}) \rightarrow H^{2}(M, \mathbb{C})
$$

and then to use the computer program SINGULAR to list the irreducible components of the determinantal variety corresponding to $R_{1}(d \mathcal{A})$; for details see [10].

For each $f_{E}$ in the list above we can use the method described in [5] to show that there is no translated component in $\mathcal{V}_{1}(d \mathcal{A})$ associated to such an $f_{E}$.

It was discovered by A. Suciu (again by using computer computations) that $\mathcal{V}_{1}(d \mathcal{A})$ has one one-dimensional translated component $W$ associated with the mapping $f$ : $M \rightarrow \mathbb{C}^{*}$ defined in affine coordinates as

$$
f(x, y)=\frac{x(y-1)(x-y-1)^{2}}{(x-1) y(x-y+1)^{2}}
$$


and with

$$
\rho_{W}=(1,-1,-1,-1,1,1,1) \in\left(\mathbb{C}^{*}\right)^{7} .
$$

(See $[13,14]$.$) In other words,$

$$
W=\rho_{W} \cdot\left\{\left(t, t^{-1}, 1, t^{-1}, t, t^{2}, t^{-2}\right) \mid t \in \mathbb{C}^{*}\right\} .
$$

Let $V_{i}$ be the component of $\mathcal{V}_{1}(d \mathcal{A})$ corresponding to each $E_{i}$ for $i=1, \ldots, 12$, that is, $V_{i}=\exp \left(E_{i}\right)$. Then it is known $[13,14]$ that

$$
W \cap V_{8} \cap V_{9} \cap V_{10}=\rho_{W}
$$

and

$$
W \cap V_{10} \cap V_{11} \cap V_{12}=\rho_{W}^{\prime},
$$

where

$$
\rho_{W}^{\prime}=(-1,1,-1,1,-1,1,1) \in\left(\mathbb{C}^{*}\right)^{7} .
$$

Since these results were obtained as a result of computer computations, it is useful to provide a direct proof based on Corollary 3.5.

Let $A=E_{8}^{0}$ and $B=E_{9}^{0}$ be the primitive lattices in $H^{1}(M, \mathbb{Z})$ introduced above and apply the construction explained in Section 2 to them with $L=H^{1}(M, \mathbb{Z})$. Here $n=7, a=b=2$. The basis $e_{1}^{\prime}, \ldots, e_{5}^{\prime}$ can be chosen as given by the following equivalence classes

$$
e_{1}^{\prime}=\left[e_{1}-e_{3}-e_{4}+e_{6}\right], \quad e_{2}^{\prime}=\left[e_{3}\right], \quad e_{3}^{\prime}=\left[e_{2}\right], \quad e_{4}^{\prime}=\left[e_{5}\right], \quad e_{5}^{\prime}=\left[e_{7}\right] .
$$

Then $m=1$ and $d_{2}=2$. Let $f_{1}=e_{2}+e_{3}-e_{5}-e_{6}$ and $f_{2}=e_{4}-e_{2}-e_{3}$. Then

$$
\mathcal{B}=\left\{e_{1}-e_{3}-e_{4}+e_{6}, e_{3}, e_{2}, e_{5}, e_{7}, f_{1}, f_{2}\right\}
$$

is a $\mathbb{Z}$-basis of $L$ (the coefficient matrix is unimodular) and we can take

$$
g_{1}=e_{1}-e_{3}-e_{4}+e_{6} \text { and } g_{2}=2 e_{3}+f_{2} .
$$

Therefore

$$
\operatorname{Tors}\left(H^{1}(M, \mathbb{Z}) /\left(E_{8}^{0}+E_{9}^{0}\right)\right)=\mathbb{Z}_{2} .
$$

Now, by the morphism $\theta: \mathbb{Z}_{2} \rightarrow V_{8} \cap V_{9}$ used in Section 2,

$$
\hat{1} \mapsto \exp _{L}\left(\frac{1}{2}\left(g_{2}\right)\right)=\exp _{L}\left(\frac{1}{2}\left(-e_{2}+e_{3}+e_{4}\right)\right)=(1,-1,-1,-1,1,1,1)=\rho_{W} \text {. }
$$

By Corollary 3.5, it follows that

$$
V_{8} \cap V_{9}=\theta\left(\mathbb{Z}_{2}\right)=\left\{1, \rho_{W}\right\} .
$$

In exactly the same way one can show that $V_{8} \cap V_{10}=V_{9} \cap V_{10}=\left\{1, \rho_{W}\right\}$. Since clearly $\rho_{W} \in W$, it follows that the four components $V_{8}, V_{9}, V_{10}$ and $W$ meet exactly in one point.

Similarly one can show that $W \cap V_{10} \cap V_{11} \cap V_{12}=\rho_{W}^{\prime}$ and that all of the other intersections of two irreducible components are trivial. 


\section{References}

[1] D. Arapura, 'Geometry of cohomology support loci for local systems. I', J. Algebraic Geom. 6 (1997), 563-597.

[2] A. Beauville, 'Annulation du $H^{1}$ pour les fibrés en droites plats', in: Complex Algebraic Varieties (Bayreuth, 1990), Lecture Notes in Mathematics, 1507 (Springer, Berlin, 1992), pp. 1-15.

[3] D. C. Cohen and P. Orlik, 'Arrangements and local systems', Math. Res. Lett. 7 (2000), 299-316.

[4] D. C. Cohen and A. I. Suciu, 'Characteristic varieties of arrangements', Math. Proc. Cambridge Philos. Soc. 127 (1999), 33-54.

[5] A. Dimca, 'Pencils of plane curves and characteristic varieties', Preprint, 2006, math/0606442.

[6] A. Dimca, S. Papadima and A. Suciu, 'Formality, Alexander invariants, and a question of Serre', Preprint, 2005, math/0512480.

[7] — 'Alexander polynomials: essential variables and multiplicities', Int. Math. Res. Not. 2008 (2008), 36.

[8] H. Esnault, V. Schechtman and E. Viehweg, 'Cohomology of local systems on the complement of hyperplanes', Invent. Math. 109 (1992), 557-561. Erratum, Invent. Math. 112 (1993) 447.

[9] A. Libgober, 'First order deformations for rank one local systems with a non-vanishing cohomology', Topology Appl. 118(1-2) (2002), 159-168.

[10] S. Nazir, 'Hyperplane arrangements', PhD Thesis, Abdus Salam School of Mathematical Sciences, GC University Lahore, 2007.

[11] P. Orlik and H. Terao, Arrangements of Hyperplanes (Springer, Berlin, 1992).

[12] C. Simpson, 'Subspaces of moduli spaces of rank one local systems', Ann. Sci. École Norm. Sup. 26(3) (1993), 361-401.

[13] A. Suciu, 'Translated tori in the characteristic varieties of complex hyperplane arrangements', Topology Appl. 118(1-2) (2002), 209-223.

[14] _ 'Fundamental groups of line arrangements: enumerative aspects', in: Advances in Algebraic Geometry Motivated by Physics (Lowell, MA, 2000), Contemporary Mathematics, 276 (American Mathematical Society, Providence, RI, 2001), pp. 43-79.

\section{SHAHEEN NAZIR, Abdus Salam School of Mathematical Sciences, Government College University, Lahore, Pakistan \\ e-mail: shaheen.nazeer@gmail.com}

